\title{
Formation postgraduée et situation pro- fessionnelle vues par les médecins-assistants
}

\section{Un sondage sur la formation postgraduée a été mené à nouveau en 2005} auprès des médecins-assistants qui briguent un titre de formation postgrade fédéral. Le taux de réponses a été de $68 \%$. La part de médecins-assistants titulaires d'un diplôme fédéral a une nouvelle fois reculé en 2005. Il apparaît manifestement qu'en Suisse, on ne forme pas suffisamment de médecins pour couvrir les besoins. Les tendances de ces dernières années annoncent en outre une future pénurie de spécialistes en médecine générale, ainsi qu'en psychiatrie et psychothérapie. En ce qui concerne la transmission des compétences «conduite d'entretien» et «information du patient sur son état de santé», de nettes divergences existent parfois entre les disciplines. Les temps de travail hebdomadaires, quant à eux, sont inférieurs à ceux des années précédentes (durée médiane: $52 \mathrm{~h}$ ). Deux tiers des médecins-assistants ne pensent pas que cette réduction du temps de travail a des effets négatifs sur leur formation postgraduée.

\section{Michael Siegrist ${ }^{a}$,}

Pascale Orlow ${ }^{a}$, Max Giger ${ }^{b}$

a Centre de recherche sociale de l’Université de Zurich

b Département de la formation médicale de la FMH

1 Siegrist M, Orlow P, Giger M. Weiterbildung aus der Sicht der Assistenzärzte: Die wichtigsten Resultate der Umfrage 2004 bei Assistenzärztinnen und -ärzten über die Weiterbildung. Schweiz Ärztezeitung 2005;86:412-23.

2 Goldacre MJ, Lambert T, Evans J, Turner G. Preregistration house officers' views on whether their experience at medical school prepared them well for their jobs: National questionnaire survey. BMJ. 2003;326:1011-2.

Correspondance:

Dr Michael Siegrist, p.-d.

Centre de recherche sociale

de l'Université de Zurich

Plattenstrasse 14

CH-8032 Zurich
L'enquête menée en 2005 auprès des médecinsassistants livre une image complète sur la manière dont ils évaluent leur formation et leur situation professionnelle. Ce sondage annuel s'inscrit dans le processus d'assurance-qualité de la formation postgraduée. De plus, il permet de déceler précocement les tendances qui se dessinent dans ce domaine.

Le questionnaire du sondage 2004 [1] a été grandement remanié. Le Comité consultatif (ou Advisory Board) instauré au printemps 2005 a joué un rôle important dans ce remaniement. Les sociétés de discipline les plus importantes sont représentées au sein de ce comité. Ses membres se rencontrent deux fois par année et participent aux décisions concernant les priorités à poser dans l'établissement du questionnaire et la manière d'informer les dirigeants des établissements de formation postgraduée sur les résultats du sondage.

L'enquête 2005 accorde une plus grande importance aux divers contenus d'enseignement transmis que les années précédentes. Concrètement, on a cherché par ex. à cerner la contribution des établissements de formation au développement des différentes compétences, aptitudes et connaissances. L'objectif était moins de connaître l'opinion subjective des personnes concernées sur leur formation postgraduée que de se renseigner sur les contenus effectivement transmis. Malgré les modifications ainsi apportées, l'appréciation de la qualité de la formation postgraduée par les médecins-assistants reste forcément subjective. Toutefois, en médecine comme ailleurs, il arrive souvent que l'on procède, pour des raisons économiques, à une appréciation de la qualité fondée sur des données émanant de sondages [2]. Une série de questions sur la situation professionnelle a constitué un autre point fort du sondage 2005. Elles devaient servir à juger de l'influence que la nouvelle loi sur le travail exerce, selon les médecins-assistants, sur la formation postgraduée. Ces questions avaient également été souhaitées par le Comité consultatif.

Des questions spécifiques ont été posées, de surcroît, à l'intention des sociétés de spécialistes en psychiatrie et psychothérapie ainsi qu'en chirurgie orthopédique et traumatologie de l'appareil locomoteur. Les sociétés de discipline étaient responsables de la formulation et du choix desdites questions.

Cette neuvième enquête sur la formation postgraduée des médecins-assistants a eu lieu au 
cours des mois de juillet à novembre 2005. Les responsables des établissements de formation postgraduée ont reçu un retour d'information détaillé avec les résultats de leur établissement et les valeurs de référence des autres établissements de la même discipline.

Le présent article se concentre sur les nouvelles parties du questionnaire. De plus, les résultats de l'enquête 2005 seront comparés avec de précédents sondages sur certains points choisis.

\section{Méthode}

\section{Ensemble de personnes sondées}

Les questionnaires ont été adressés à 1197 dirigeants d'établissements de formation postgraduée qui se sont chargés de les distribuer à 8447 médecins-assistants. Ces derniers pouvaient envoyer directement leurs questionnaires remplis au Centre de recherche sociale de l'Université de Zurich, responsable de l'enquête. Le taux de participation a été de $68 \%(\mathrm{~N}=5751)$. Il est comparable au taux de participation des enquêtes 2003 et 2004 [1, 3]. 48\% des questionnaires ont été remplis par des assistants femmes et $52 \%$ par des hommes. Concernant l'appréciation de la situation au travail, seules ont été prises en considération les réponses des médecins-assistants ayant occupé une même place pendant au moins deux mois.

3 Siegrist M, Gutscher H, Giger M. Was haben Betriebskultur, Motivation und Fremdjahr mit der Weiterbildung zu tun? Schweiz Ärztezeitung 2004;85:783-91.

\section{Questionnaire}

Le questionnaire contenait 88 items. Les sondés devaient avant tout prendre position sur un cer-

\begin{tabular}{|c|c|c|c|}
\hline \multicolumn{4}{|c|}{$\begin{array}{l}\text { Origine des médecins-assistants pour les années } 2003 \text { à 2005. Ré } \\
\text { «Dans quel pays avez-vous passé vos examens de fin d'étude?». }\end{array}$} \\
\hline & 2003 & 2004 & 2005 \\
\hline Suisse & $67,8 \%(n=3561)$ & $64,7 \%(n=3436)$ & $62,7 \%(n=3555)$ \\
\hline Etranger & $32,2 \%(n=1692)$ & $35,3 \%(n=1876)$ & $37,3 \%(n=2116)$ \\
\hline
\end{tabular}

Tableau 2

Titres de spécialistes visés pour les années 2003 à 2005: médecins-assistants briguant un titre de spécialiste en médecine générale.

$\begin{array}{lccc} & 2003 & 2004 & 2005 \\ \text { Médecine générale } & 9,2 \%(n=444) & 8,5 \%(n=426) & 8,0 \%(n=427) \\ \text { Autres titres de spécialistes } & 90,8 \%(n=4373) & 91,5 \%(n=4585) & 92,0 \%(n=4903)\end{array}$

Remarque: sont indiqués dans chaque colonne les valeurs absolues et les pourcentages respectifs. tain nombre d'affirmations. Pour la plupart des celles-ci, six réponses étaient possibles, allant de 1 «ce n'est absolument pas exact» à 6 «c'est parfaitement exact».

\section{Résultats}

Le nombre de médecins-assistants qui ont un diplôme officiel étranger a augmenté ces dernières années. Si, en 2003, seuls $32 \%$ des médecinsassistants n'avaient pas passé l'examen d'Etat en Suisse, ils étaient déjà 37\% en 2005 (tab. 1).

Le tableau 2 indique combien de médecinsassistants souhaitent obtenir le titre de spécialiste en médecine générale. Les données de ces trois dernières années révèlent une tendance à la baisse. En 2005, seuls 8\% des sondés avaient encore l'intention de devenir généralistes. Des analyses complémentaires ont montré que les différences entre les diverses régions du pays ne sont pas significatives. Autrement dit, dans toutes les régions, il y a un recul du nombre des médecinsassistants se préparant pour le titre de spécialiste en médecine générale.

Le tableau 3 montre les titres de spécialistes visés par les candidats des cohortes 1998 à 2004. Des résultats très similaires à ceux de l'enquête 2004 ont été observés [1]. Une tendance négative a été relevée pour la psychiatrie et la psychothérapie. Le chiffre relatif des médecins-assistants se préparant pour ce titre est nettement plus bas dans les cohortes 2003 et 2004 que dans les cohortes de 1998 à 2000. La tendance contraire observée chez les chirurgiens s'est maintenue. Comme auparavant, la part relative des chirurgiens au sein des nouvelles cohortes est nettement plus élevée que dans les plus anciennes.

\section{Transmission des compétences professionnelles}

Les médecins-assistants ont été interrogés sur la contribution fournie par les établissements de formation postgraduée au développement de diverses compétences, aptitudes et connaissances. Les résultats figurent dans le tableau 4 ci-après. De très hautes valeurs ont été attribuées aux connaissances professionnelles spécifiques à la discipline, à l'activité indépendante et la prise de responsabilité. Selon les médecins-assistants, la transmission de ces connaissances et compétences fonctionne très bien dans la plupart des établissements de formation postgraduée.

La valeur la plus basse a été attribuée à l'accompagnement des mourants. Une partie des assistants ne se sentent pas bien préparés à cette tâche. Des valeurs basses ont également été enregistrées en ce qui concerne l'appréciation de la 
Tableau 3

Cohortes des titulaires d'un diplôme de médecin obtenu entre 1998 et 2004 et titres de spécialistes visés.

\begin{tabular}{|c|c|c|c|c|c|c|c|}
\hline & 1998 & 1999 & 2000 & 2001 & 2002 & 2003 & 2004 \\
\hline Médecine générale & $7,0 \%(n=30)$ & $8,3 \%(n=48)$ & $7,9 \%(n=47)$ & $9,0 \%(n=65)$ & $8,1 \%(n=58)$ & $5,4 \%(n=35)$ & $7,5 \%(n=48)$ \\
\hline Anesthésiologie & $9,1 \%(n=39)$ & $8,0 \%(n=46)$ & $5,7 \%(n=34)$ & $6,6 \%(n=48)$ & $7,1 \%(n=51)$ & $5,2 \%(n=34)$ & $5,3 \%(n=34)$ \\
\hline Chirurgie & $2,6 \%(n=11)$ & $4,3 \%(n=25)$ & $5,2 \%(n=31)$ & $6,9 \%(n=50)$ & $7,8 \%(n=56)$ & $8,9 \%(n=58)$ & $8,6 \%(n=55)$ \\
\hline Gynécologie & $7,2 \%(n=31)$ & $8,7 \%(n=50)$ & $6,2 \%(n=37)$ & $6,3 \%(n=46)$ & $6,4 \%(n=46)$ & $7,1 \%(n=46)$ & $7,2 \%(n=46)$ \\
\hline Médecine interne & $12,9 \%(n=55)$ & $16,4 \%(n=95)$ & $19,6 \%(n=117)$ & $22,6 \%(n=164)$ & $24,9 \%(n=178)$ & $20,2 \%(n=131)$ & $15,7 \%(n=100)$ \\
\hline Pédiatrie & $6,5 \%(n=28)$ & $8,1 \%(n=47)$ & $6,5 \%(n=39)$ & $7,6 \%(n=55)$ & $6,2 \%(n=44)$ & $7,1 \%(n=46)$ & $9,2 \%(n=59)$ \\
\hline $\begin{array}{l}\text { Chirurgie orthopédique } \\
\text { et traumatologie } \\
\text { de l'appareil locomoteur }\end{array}$ & $3,7 \%(n=16)$ & $4,8 \%(n=28)$ & $6,5 \%(n=39)$ & $6,1 \%(n=44)$ & $4,5 \%(n=32)$ & $6,8 \%(n=44)$ & $6,0 \%(n=38)$ \\
\hline $\begin{array}{l}\text { Psychiatrie et } \\
\text { psychothérapie }\end{array}$ & $12,4 \%(n=53)$ & $11,1 \%(n=64)$ & $11,7 \%(n=70)$ & $7,3 \%(n=53)$ & $6,3 \%(n=45)$ & $5,5 \%(n=36)$ & $4,2 \%(n=27)$ \\
\hline $\begin{array}{l}\text { Autres / pas encore } \\
\text { de décision }\end{array}$ & $38,6 \%(n=165)$ & $30,3 \%(n=175)$ & $30,7 \%(n=183)$ & $27,7 \%(n=201)$ & $28,6 \%(n=204)$ & $33,7 \%(n=219)$ & $36,2 \%(n=231)$ \\
\hline
\end{tabular}

Remarque: sont indiqués dans chaque colonne les valeurs absolues et les pourcentages respectifs.

\section{Tableau 4}

Valeurs moyennes $(M)$, écarts standards $(S D)$ et nombre de réponses valables $(N)$ pour les questions concernant la contribution des établissements de formation postgraduée au développement de diverses compétences, aptitudes et connaissances.

\begin{tabular}{|c|c|c|c|}
\hline Aspects considérés & M & SD & $\mathrm{N}$ \\
\hline Activité indépendante & 5,06 & 1,01 & 5394 \\
\hline Connaissances professionnelles spécifiques à la discipline & 5,04 & 0,93 & 5376 \\
\hline Collaboration avec le personnel infirmier & 5,00 & 1,01 & 5107 \\
\hline Prise de responsabilités & 4,91 & 1,05 & 5399 \\
\hline Information claire et compréhensible du patient sur son état de santé & 4,91 & 1,05 & 5140 \\
\hline Pose d'indication pour des traitements & 4,89 & 0,99 & 5125 \\
\hline Application pratique du savoir théorique & 4,88 & 1,01 & 5370 \\
\hline Information du patient sur les risques et l'utilité d'une intervention & 4,87 & 1,07 & 4820 \\
\hline Raisonnement clinique & 4,86 & 1,04 & 5315 \\
\hline Etablissement de rapports médicaux & 4,85 & 1,09 & 5124 \\
\hline Collaboration avec d'autres disciplines médicales & 4,79 & 1,02 & 5343 \\
\hline Interprétation des résultats d'examens & 4,79 & 1,03 & 5153 \\
\hline Collaboration avec des services paramédicaux & 4,73 & 1,15 & 4841 \\
\hline Pose d'indication pour des examens spéciaux & 4,71 & 1,09 & 5200 \\
\hline Evaluation réaliste de ses propres capacités & 4,70 & 1,00 & 5348 \\
\hline Utilisation correcte de médicaments (pharmacothérapie efficace et sûre) & 4,67 & 1,11 & 5145 \\
\hline Anamnèse & 4,66 & 1,21 & 5148 \\
\hline Application de nouvelles connaissances scientifiques & 4,62 & 1,17 & 5320 \\
\hline Collaboration avec les médecins / médecins de premier recours chargés du suivi & 4,60 & 1,11 & 5048 \\
\hline $\begin{array}{l}\text { Maîtrise des techniques d'intervention et d'examen clinique (p.ex. opérations, examens cliniques, } \\
\text { examens spéciaux, entretiens) }\end{array}$ & 4,56 & 1,21 & 4758 \\
\hline Principes éthiques de la médecine & 4,56 & 1,21 & 5212 \\
\hline Techniques d'examen clinique & 4,46 & 1,24 & 5040 \\
\hline Conduite d'entretien & 4,43 & 1,29 & 5112 \\
\hline Analyse utilité-risque dans le cadre des diagnostics et des traitements & 4,40 & 1,25 & 5025 \\
\hline Analyse des erreurs & 4,37 & 1,29 & 5324 \\
\hline $\begin{array}{l}\text { Utilisation économique des ressources dans le cadre des } \\
\text { diagnostics et des traitements }\end{array}$ & 4,31 & 1,26 & 5180 \\
\hline Evaluation de la capacité de travail du patient & 4,07 & 1,37 & 4203 \\
\hline Accompagnement de mourants & 3,77 & 1,60 & 3523 \\
\hline
\end{tabular}

Remarque: les valeurs attribuées oscillent entre 1 «très peu» et 6 «énormément». Seules ont été prises en compte les réponses de médecins-assistants ayant exercé une activité dans un établissement de formation postgraduée pendant au moins deux mois. Lorsqu'un aspect ne concernait pas une discipline donnée, il était possible de cocher l'option «non applicable». Ces dernières valeurs n'ont pas été incluses dans le calcul des valeurs moyennes et des écarts standards. 
capacité de travail des patients, l'utilisation économique des ressources à disposition ou l'analyse des erreurs.

Les mêmes compétences ne sont pas transmises dans toutes les disciplines. Le tableau 5 montre qu'il existe de nettes différences en ce qui concerne la transmission de ces compétences dans les sept disciplines comprenant le plus de médecins-assistants. Dans les domaines de l'anesthésiologie, de la chirurgie, de la chirurgie orthopédique et de la traumatologie de l'appareil locomoteur, des valeurs basses ont été attribuées à la transmission des compétences en matière de conduite d'entretien et d'information des patients sur leur état de santé.

\section{Situation professionnelle}

Pour une majorité de médecins-assistants, la nouvelle loi sur le travail ne semble pas avoir une influence négative sur la formation postgraduée et le travail (tab. 6). Cela dit, un médecin-assistant sur quatre a l'impression de ne pas pouvoir accomplir son activité à son entière satisfaction dans le cadre du temps de travail fixé par contrat. La plupart des établissements de formation semblent disposer de l'infrastructure nécessaire à un travail efficace. La situation sur le plan des contraintes administratives a été moins favora- blement évaluée. Près de la moitié des sondés ont le sentiment que les travaux administratifs les entravent dans leur activité auprès du patient ou dans leur formation postgraduée. L'influence de la loi sur le travail sur la formation postgraduée et sur l'activité au chevet du patient a été appréciée de manières diverses. Alors que sept médecins-assistants sur dix ne voient pas d'effets tendanciellement négatifs découlant de cette nouvelle loi, trois assistants sur dix pensent en revanche qu'elle a des effets plus ou moins négatifs sur la formation postgraduée. Des résultats similaires ont été obtenus sur la question des effets de la loi sur le travail sur l'activité au chevet du patient. Néanmoins, seule une petite partie des médecins-assistants a l'impression que le temps de travail imposé par la loi agit négativement sur la formation postgraduée et le travail auprès du patient.

Les plus grandes différences entre les disciplines ont été observées dans les réactions à l'affirmation «Les travaux administratifs entravent ma formation postgraduée». Le tableau 7 ciaprès présente les valeurs moyennes obtenues pour les sept disciplines comprenant le plus de médecins-assistants. Les moins touchés par les tâches administratives sont celles et ceux qui travaillent en anesthésiologie. Viennent ensuite les

\begin{tabular}{|c|c|c|c|}
\hline \multicolumn{4}{|c|}{$\begin{array}{l}\text { Valeurs moyennes pour la transmission de diverses compétences dans les sept disciplines comprenant le plus } \\
\text { de médecins-assistants (IC } 95 \%=\text { intervalle de confiance à } 95 \%) .\end{array}$} \\
\hline \multicolumn{4}{|l|}{ Conduite d'entretien } \\
\hline Discipline & Valeur moyenne & IC $95 \%$ & $\mathrm{~N}$ \\
\hline Anesthésiologie & 3,24 & $3,04-3,43$ & 219 \\
\hline Chirurgie & 3,93 & $3,83-4,03$ & 649 \\
\hline Chirurgie orthopédique et traumatologie de l'appareil locomoteur & 3,95 & $3,77-4,13$ & 211 \\
\hline Pédiatrie & 4,42 & $4,29-4,56$ & 264 \\
\hline Médecine interne & 4,59 & $4,53-4,66$ & 1306 \\
\hline Gynécologie et obstétrique & 4,60 & $4,47-4,72$ & 324 \\
\hline Psychiatrie et psychothérapie & 4,77 & $4,68-4,85$ & 689 \\
\hline \multicolumn{4}{|l|}{ Information claire et compréhensible du patient sur son état de santé } \\
\hline Discipline & Valeur moyenne & IC $95 \%$ & $\mathrm{~N}$ \\
\hline Anesthésiologie & 4,23 & $4,05-4,42$ & 201 \\
\hline Chirurgie & 4,67 & $4,59-4,76$ & 657 \\
\hline Chirurgie orthopédique et traumatologie de l'appareil locomoteur & 4,74 & $4,59-4,89$ & $21 \varepsilon$ \\
\hline Pédiatrie & 5,06 & $4,96-5,17$ & 263 \\
\hline Médecine interne & 4,97 & $4,92-5,02$ & 1321 \\
\hline Gynécologie et obstétrique & 5,11 & $5,01-5,21$ & 327 \\
\hline Psychiatrie et psychothérapie & 5,01 & $4,93-5,08$ & 690 \\
\hline
\end{tabular}

Remarque: les valeurs attribuées oscillent entre 1 «très peu» et 6 «énormément». Seules ont été prises en compte les réponses de médecins-assistants ayant exercé une activité dans un établissement de formation postgraduée pendant au moins deux mois. 
médecins-assistants en psychiatrie et psychothérapie et en gynécologie et obstétrique, dont la formation postgraduée est relativement peu influencée par les travaux administratifs. Les plus concernés sont les médecins-assistants dans les disciplines chirurgicales, la pédiatrie, la chirurgie orthopédique et la traumatologie de l'appareil locomoteur, ainsi que la médecine interne.
Depuis le $1^{\text {er }}$ janvier 2005, les médecinsassistants sont soumis à la loi sur le travail. Le tableau 8 montre que seule une minorité d'entre eux indiquent ne jamais rester au travail plus longtemps que 14 heures d'affilée. De nettes différences peuvent être observées entre les disciplines. C'est en chirurgie orthopédique et traumatologie de l'appareil locomoteur ainsi qu'en

\section{Tableau 6}

Pourcentages respectifs concernant sept questions sur la situation professionnelle.

\begin{tabular}{|c|c|c|c|c|c|}
\hline $\begin{array}{l}1 \text { (ce n'est absolument pas } \\
\text { exact) }\end{array}$ & 2 & 3 & 4 & 5 & $\begin{array}{l}6 \text { (c'est parfaitement } \\
\text { exact) }\end{array}$ \\
\hline
\end{tabular}

La durée de travail définie dans mon contrat me permet d'accomplir mon travail à mon entière satisfaction.

$\begin{array}{llllll}4,4 \% & 8,5 \% & 9,7 \% & 19,0 \% & 33,1 \% & 25,3 \%\end{array}$

Je dispose de l'infrastructure nécessaire (p.ex. poste de travail muni d'un ordinateur, accès à l'internet) et peux accomplir mon travail avec efficacité.

$\begin{array}{llllll}1,6 \% & 2,8 \% & 5,7 \% & 11,7 \% & 29,6 \% & 48,6 \%\end{array}$

Les travaux administratifs entravent ma formation postgraduée.

$\begin{array}{llllll}12,1 \% & 18,7 \% & 12,5 \% & 19,6 \% & 22,2 \% & 15,0 \%\end{array}$

Les travaux administratifs entravent mon activité au chevet du patient.

$\begin{array}{llllll}14,5 \% & 19,0 \% & 11,4 \% & 19,2 \% & 21,1 \% & 14,8 \%\end{array}$

Je suis informé suffisamment tôt des décisions importantes prises par la direction de l'hôpital qui influent sur ma formation postgraduée.

$\begin{array}{llllll}8,3 \% & 11,1 \% & 16,0 \% & 23,2 \% & 25,2 \% & 16,2 \%\end{array}$

La réglementation par la loi de la durée du temps de travail a une incidence négative sur ma formation postgraduée.
$35,4 \%$
$23,5 \%$
$10,8 \%$
$12,4 \%$
$9,9 \%$
$8,0 \%$

La réglementation par la loi de la durée du temps de travail a une répercussion négative sur mon activité au chevet du patient. $\begin{array}{llllll}40,4 \% & 25,6 \% & 10,9 \% & 10,8 \% & 7,7 \% & 4,7 \%\end{array}$

Remarque: $\mathrm{N}=5233$ à 5404 (différences dues aux valeurs manquantes). Seules ont été prises en compte les réponses de médecins-assistants ayant exercé une activité dans un établissement de formation postgraduée pendant au moins deux mois.

Tableau 7

Valeurs moyennes concernant l'affirmation «Les travaux administratifs entravent ma formation postgraduée» pour les sept disciplines comprenant le plus de médecins-assistants.

\begin{tabular}{lllr} 
Discipline & Valeur moyenne & IC $95 \%$ & N \\
\hline Anesthésiologie & 2,18 & $2,02-2,35$ & 250 \\
\hline Psychiatrie et psychothérapie & 3,48 & $3,35-3,60$ & 688 \\
\hline Gynécologie et obstétrique & 3,74 & $3,57-3,91$ & 328 \\
\hline Chirurgie & 4,04 & $3,92-4,16$ & 662 \\
\hline Pédiatrie & 4,10 & $3,92-4,27$ & 265 \\
\hline Chirurgie orthopédique et traumatologie de I'appareil locomoteur & 4,11 & $3,91-4,31$ & 221 \\
\hline Médecine interne & 4,13 & $4,04-4,21$ & 1310
\end{tabular}

Remarque: les valeurs attribuées oscillent entre 1 «très peu» et 6 «énormément». Seules ont été prises en compte les réponses de médecins-assistants ayant exercé une activité dans un établissement de formation postgraduée pendant au moins deux mois. 
chirurgie que l'on éprouve le plus de difficultés à s'adapter aux nouvelles règles. En revanche, les nouvelles dispositions sont mieux suivies dans les domaines de la psychiatrie et psychothérapie et de l'anesthésiologie. Des différences semblables entre les disciplines ont été enregistrées à la question «A quelle fréquence n'êtes-vous pas en mesure de respecter le temps de repos prévu par la loi?» (tab. 9).

Le tableau 10 présente les réponses à la question de savoir si plus de 6 jours de service sont accomplis d'affilée. C'est uniquement dans le domaine de la psychiatrie et psychothérapie qu'une majorité de médecins-assistants décla- rent ne jamais travailler plus de 6 périodes de service de suite.

Une majorité des médecins-assistants indiquent par ailleurs que les 50 heures de travail par semaine sont respectées (tab. 11). Mais pour cette question également, des différences significatives existent entre les disciplines. Selon l'estimation des médecins-assistants, les prescriptions sont bien appliquées en psychiatrie et psychothérapie ainsi qu'en anesthésiologie. Les plus grands problèmes d'application des nouvelles prescriptions semblent toucher la chirurgie orthopédique, la traumatologie de l'appareil locomoteur et l'anesthésiologie.

Tableau 8

Réponses à la question «A quelle fréquence vous arrive-t-il de travailler plus de 14 heures à la suite, durée maximale prévue par la loi?» dans les sept disciplines comprenant le plus de médecins-assistants.

\begin{tabular}{|c|c|c|c|c|c|c|}
\hline & jamais & $\begin{array}{l}\text { très } \\
\text { rarement }\end{array}$ & $\begin{array}{l}\text { de temps } \\
\text { en temps }\end{array}$ & souvent & $\begin{array}{l}\text { régulière- } \\
\text { ment }\end{array}$ & total \\
\hline $\begin{array}{l}\text { Chirurgie orthopédique } \\
\text { et traumatologie } \\
\text { de I'appareil locomoteur }\end{array}$ & $6,8 \%$ & $42,3 \%$ & $38,6 \%$ & $8,2 \%$ & $4,1 \%$ & $100 \%(n=220)$ \\
\hline Chirurgie & $11,3 \%$ & $38,8 \%$ & $38,7 \%$ & $8,8 \%$ & $2,4 \%$ & $100 \%(n=662)$ \\
\hline $\begin{array}{l}\text { Gynécologie et } \\
\text { obstétrique }\end{array}$ & $14,3 \%$ & $43,6 \%$ & $32,9 \%$ & $6,4 \%$ & $2,7 \%$ & $100 \%(n=328)$ \\
\hline Médecine interne & $21,3 \%$ & $42,7 \%$ & $28,4 \%$ & $5,5 \%$ & $2,2 \%$ & $100 \%(n=1326)$ \\
\hline Pédiatrie & $17,4 \%$ & $35,6 \%$ & $35,6 \%$ & $7,2 \%$ & $4,2 \%$ & $100 \%(n=264)$ \\
\hline Anesthésiologie & $37,3 \%$ & $42,6 \%$ & $16,1 \%$ & $1,6 \%$ & $2,4 \%$ & $100 \%(n=249)$ \\
\hline $\begin{array}{l}\text { Psychiatrie et } \\
\text { psychothérapie }\end{array}$ & $43,9 \%$ & $36,5 \%$ & $16,3 \%$ & $2,8 \%$ & $0,6 \%$ & $100 \%(n=688)$ \\
\hline
\end{tabular}

Remarque: seules ont été prises en compte les réponses de médecins-assistants ayant exercé une activité dans un établissement de formation postgraduée pendant au moins deux mois. $\chi_{(24)}^{2}=377,73, p<0,001$.

\begin{tabular}{|c|c|c|c|c|c|c|}
\hline & jamais & $\begin{array}{l}\text { très } \\
\text { rarement }\end{array}$ & $\begin{array}{l}\text { de temps } \\
\text { en temps }\end{array}$ & souvent & $\begin{array}{l}\text { régulière- } \\
\text { ment }\end{array}$ & total \\
\hline $\begin{array}{l}\text { Chirurgie orthopédique } \\
\text { et traumatologie } \\
\text { de I'appareil locomoteur }\end{array}$ & $10,2 \%$ & $38,4 \%$ & $33,3 \%$ & $11,6 \%$ & $6,5 \%$ & $100 \%(n=216)$ \\
\hline Chirurgie & $13,1 \%$ & $41,0 \%$ & $28,4 \%$ & $12,9 \%$ & $4,6 \%$ & $100 \%(n=651)$ \\
\hline $\begin{array}{l}\text { Gynécologie et } \\
\text { obstétrique }\end{array}$ & $21,2 \%$ & $34,5 \%$ & $29,5 \%$ & $8,9 \%$ & $5,8 \%$ & $100 \%(n=325)$ \\
\hline Médecine interne & $25,7 \%$ & $37,4 \%$ & $24,4 \%$ & $8,3 \%$ & $4,3 \%$ & $100 \%(n=1301)$ \\
\hline Pédiatrie & $18,9 \%$ & $39,8 \%$ & $26,3 \%$ & $8,9 \%$ & $6,2 \%$ & $100 \%(n=259)$ \\
\hline Anesthésiologie & $31,3 \%$ & $39,3 \%$ & $17,9 \%$ & $6,0 \%$ & $5,6 \%$ & $100 \%(n=252)$ \\
\hline $\begin{array}{l}\text { Psychiatrie et } \\
\text { psychothérapie }\end{array}$ & $45,2 \%$ & $30,7 \%$ & $16,8 \%$ & $4,2 \%$ & $2,9 \%$ & $100 \%(n=683)$ \\
\hline
\end{tabular}

Remarque: seules ont été prises en compte les réponses de médecins-assistants ayant exercé une activité dans un établissement de formation postgraduée pendant au moins deux mois. $\chi_{(24)}^{2}=263,06, p<0,001$. 


\section{Tableau 10}

Réponses à l'affirmation «Je ne travaille jamais plus de 6 jours de service d'affilée» dans les sept disciplines comprenant le plus de médecins-assistants.

\begin{tabular}{llll} 
& oui & non & total \\
\hline $\begin{array}{lll}\text { Chirurgie orthopédique et traumatologie } \\
\text { de l'appareil locomoteur }\end{array}$ & $44,3 \%$ & $55,7 \%$ & $100 \%(n=219)$ \\
\hline Chirurgie & $43,1 \%$ & $56,9 \%$ & $100 \%(n=649)$ \\
\hline Gynécologie et obstétrique & $42,1 \%$ & $57,9 \%$ & $100 \%(n=328)$ \\
\hline Médecine interne & $46,8 \%$ & $53,2 \%$ & $100 \%(n=1312)$ \\
\hline Pédiatrie & $40,5 \%$ & $59,5 \%$ & $100 \%(n=262)$ \\
\hline Anesthésiologie & $45,2 \%$ & $54,8 \%$ & $100 \%(n=252)$ \\
\hline Psychiatrie et psychothérapie & $85,1 \%$ & $14,9 \%$ & $100 \%(n=677)$
\end{tabular}

Remarque: seules ont été prises en compte les réponses de médecins-assistants ayant exercé une activité dans un établissement de formation postgraduée pendant au moins deux mois. $\chi_{(6)}^{2}=368,23, p<0,001$.

\section{Tableau 11}

Réponses à l'affirmation «Le temps de travail convenu dans le contrat d'engagement (activité à $100 \%=50 \mathrm{~h} /$ semaine) est appliqué» dans les sept disciplines comprenant le plus de médecinsassistants

\begin{tabular}{|c|c|c|c|}
\hline & oui & non & total \\
\hline $\begin{array}{l}\text { Chirurgie orthopédique et traumatologie } \\
\text { de l'appareil locomoteur }\end{array}$ & $64,4 \%$ & $35,6 \%$ & $100 \%(n=216)$ \\
\hline Chirurgie & $67,7 \%$ & $32,3 \%$ & $100 \%(n=648)$ \\
\hline Gynécologie et obstétrique & $82,2 \%$ & $17,8 \%$ & $100 \%(n=320)$ \\
\hline Médecine interne & $71,6 \%$ & $28,4 \%$ & $100 \%(n=1290)$ \\
\hline Pédiatrie & $77,5 \%$ & $22,5 \%$ & $100 \%(n=253)$ \\
\hline Anesthésiologie & $87,1 \%$ & $12,9 \%$ & $100 \%(n=249)$ \\
\hline Psychiatrie et psychothérapie & $90,0 \%$ & $10,0 \%$ & $100 \%(n=681)$ \\
\hline
\end{tabular}

Remarque: seules ont été prises en compte les réponses de médecins-assistants ayant exercé une activité dans un établissement de formation postgraduée pendant au moins deux mois. $\chi_{(6)}^{2}=152,29, p<0,001$.
Le temps de travail hebdomadaire des médecins-assistants a considérablement diminué ces dernières années en raison des prescriptions légales.

La valeur médiane se situe actuellement à 52 heures. Le tableau 12 indique que dans chacune des sept disciplines avec le plus grand nombre de médecins-assistants, le travail a comporté moins d'heures en 2005 qu'en 2003. Les différences entre les disciplines n'ont cependant guère changé. En 2005 à nouveau, les valeurs moyennes de travail hebdomadaire les plus élevées ont été observées en chirurgie orthopédique et traumatologie de l'appareil locomoteur ainsi qu'en chirurgie.

La nouvelle loi ne semble pas avoir d'influence négative sur l'étendue de la formation postgraduée théorique. Le tableau 13 indique combien d'heures de formation postgraduée théorique ont été suivies en moyenne par les médecins-assistants des disciplines comprenant le plus grand nombre d'assistants. Les chiffres de l'enquête 2005 sont comparables aux valeurs obtenues les années précédentes.

\section{Discussion}

Si l'on considère les résultats des enquêtes menées auprès des médecins-assistants ces trois dernières années, deux développements retiennent tout particulièrement l'attention. Il s'agit premièrement de la part des médecins-assistants qui ont passé leur examen d'Etat à l'étranger, laquelle a constamment augmenté ces dernières années. En Suisse, on ne forme pas suffisamment de médecins-assistants pour répondre aux besoins de la situation hospitalière telle qu'elle se présente actuellement. Une deuxième tendance

Tableau 12

Valeurs moyennes relatives au temps de travail hebdomadaire moyen des médecins-assistants travaillant à temps plein dans les sept disciplines comprenant le plus de médecins-assistants.

\begin{tabular}{|c|c|c|c|c|c|c|}
\hline \multirow[b]{2}{*}{ Discipline } & \multicolumn{3}{|l|}{2003} & \multicolumn{3}{|l|}{2005} \\
\hline & M & IC $95 \%$ & $\mathrm{~N}$ & M & IC $95 \%$ & $\mathrm{~N}$ \\
\hline Chirurgie orthopédique et traumatologie de l'appareil locomoteur & 61,19 & $60,18-62,21$ & 175 & 56,73 & $55,93-57,53$ & 200 \\
\hline Chirurgie & 59,85 & $59,30-60,40$ & 587 & 56,00 & $55,50-56,50$ & 618 \\
\hline Gynécologie et obstétrique & 57,84 & $57,14-58,53$ & 285 & 53,60 & $52,94-54,26$ & 277 \\
\hline Médecine interne & 57,04 & $56,71-57,37$ & 1100 & 54,48 & $54,17-54,78$ & 1196 \\
\hline Pédiatrie & 56,85 & $56,12-57,57$ & 217 & 53,82 & $53,12-54,52$ & 227 \\
\hline Anesthésiologie & 54,41 & $53,87-54,95$ & 224 & 52,12 & $51,62-52,62$ & 239 \\
\hline Psychiatrie et psychothérapie & 52,78 & $52,28-53,28$ & 454 & 51,15 & $50,76-51,54$ & 438 \\
\hline
\end{tabular}

Remarque: seules ont été prises en compte les réponses de médecins-assistants ayant exercé une activité dans un établissement de formation postgraduée pendant au moins deux mois. Les cas extrêmes n'ont pas été pris en considération. Données en heures par semaine. 
se rapporte au nombre de médecins-assistants qui visent un titre de spécialiste en médecine générale ou en psychiatrie et psychothérapie. Leur part a de nouveau diminué en 2005. De futures carences se dessinent ici dans la couverture des soins en médecine de premier recours et en psychiatrie. Les deux tendances ont un point commun: il faut s'attendre à ce que la Suisse devienne à l'avenir encore plus dépendante de l'étranger si elle entend pouvoir maintenir, avec les structures actuelles, un système de santé en état de fonctionner.

Dans l'ensemble, la formation postgraduée fonctionne bien. En moyenne, les établissements de formation fournissent aux médecinsassistants une aide adéquate leur permettant d'acquérir des compétences importantes. Même si un bon travail est accompli à cet égard dans la plupart des établissements, certains d'entre eux n'ont pas encore exploité tout leur potentiel. Cela concerne notamment les compétences en matière d'accompagnement des mourants et d'utilisation économique des ressources à disposition ainsi que la culture de sécurité. Il y a aussi des disciplines dans lesquelles certaines compétences ne sont pas suffisamment enseignées. Ainsi, en anesthésiologie, en chirurgie, de même qu'en chirurgie orthopédique et traumatologie de l'appareil locomoteur, les compétences «conduite d'entretien» ou «information du patient» sont moins bien transmises que dans d'autres disciplines. Il existe là nettement un retard à rattraper.

Dans l'ensemble, les médecins-assistants ont jugé leur situation professionnelle de manière positive. Toutefois, la situation de travail d'une minorité que l'on ne saurait négliger devrait être améliorée. Ainsi, un médecin-assistant sur cinq est plus ou moins d'avis qu'il ne peut accomplir son travail à son entière satisfaction pendant son temps de travail réglé par contrat. Les effets de la réglementation du temps de travail sur la formation postgraduée sont appréciés de manières diverses: pour deux tiers des sondés, les directives légales en matière de temps de travail n'ont pas de conséquence négative sur la formation postgraduée. Un petit tiers est néanmoins d'avis que la nouvelle réglementation influe négativement sur sa propre formation postgraduée.

Les médecins-assistants ont, suivant les cas, nettement moins travaillé en 2005 qu'en 2003. Tous les établissements de formation postgraduée ne parviennent pas à satisfaire aux prescriptions légales. Il est heureux que malgré la réduction de la durée totale du travail, le nombre d'heures de formation postgraduée théorique suivies n'ait pas baissé. Ce sont les domaines de la psychiatrie et psychothérapie ainsi que de l'anesthésiologie qui semblent avoir pu s'adapter le mieux à la nouvelle loi sur le travail.

\section{Regard vers I'avenir}

Pour l'enquête 2006, le questionnaire sera à nouveau amélioré et optimisé. De manière à pouvoir comparer les sondages sur plusieurs années, un minimum de modifications sera apporté aux questions centrales. De nouveaux points forts, importants pour les sociétés de discipline et la FMH, seront discutés et définis d'entente avec le Comité consultatif. En effet, le questionnaire n'a pas pour seul objectif de contribuer à assurer la qualité de la formation postgraduée. Cet instrument de sondage doit aussi servir à répondre à des questions actuelles en matière de politique de formation et de santé.

Tableau 13

Valeurs moyennes relatives à la formation postgraduée théorique moyenne des médecins-assistants travaillant à temps plein dans les sept disciplines comprenant le plus de médecins-assistants.

\begin{tabular}{|c|c|c|c|c|c|c|}
\hline \multirow[b]{2}{*}{ Discipline } & \multicolumn{3}{|l|}{2003} & \multicolumn{3}{|l|}{2005} \\
\hline & M & IC $95 \%$ & $\mathrm{~N}$ & M & IC $95 \%$ & $N$ \\
\hline Anesthésiologie & 1,69 & $1,56-1,82$ & 235 & 1,78 & $1,66-1,89$ & 239 \\
\hline Gynécologie et obstétrique & 1,72 & $1,56-1,87$ & 295 & 1,76 & $1,62-1,90$ & 302 \\
\hline Chirurgie & 1,78 & $1,67-1,89$ & 619 & 1,83 & $1,73-1,93$ & 647 \\
\hline Chirurgie orthopédique et traumatologie de l'appareil locomoteur & 2,05 & $1,82-2,28$ & 182 & 2,68 & $2,39-2,96$ & 216 \\
\hline Pédiatrie & 2,42 & $2,23-2,61$ & 228 & 2,55 & $2,37-2,73$ & 240 \\
\hline Médecine interne & 2,61 & $2,52-2,70$ & 1144 & 2,68 & $2,60-2,76$ & 1269 \\
\hline Psychiatrie et psychothérapie & 4,49 & $4,31-4,68$ & 490 & 4,24 & $4,05-4,42$ & 484 \\
\hline
\end{tabular}

Remarque: seules ont été prises en compte les réponses de médecins-assistants ayant exercé une activité dans un établissement de formation postgraduée pendant au moins deux mois. Les cas extrêmes n'ont pas été pris en considération. Données en heures par semaine. 